\title{
Direct observation of particle-hole mixing in the superconducting state by angle-resolved photoemission
}

\author{
J. C. Campuzano and H. Ding \\ Materials Science Division, Argonne National Laboratory, Argonne, Illinois 60439 \\ and Department of Physics, University of Illinois at Chicago, Chicago, Illinois 60607 \\ M. R. Norman \\ Materials Science Division, Argonne National Laboratory, Argonne, Illinois 60439 \\ M. Randeira \\ Materials Science Division, Argonne National Laboratory, Argonne, Illinois 60439 \\ and Tata Institute of Fundamental Research, Bombay 400005, India \\ A. F. Bellman \\ Materials Science Division, Argonne National Laboratory, Argonne, Illinois 60439 \\ and Dipartimento di Fisica, Universitá di Milano, 20133 Milano, Italy \\ T. Yokoya, T. Takahashi, and H. Katayama-Yoshida \\ Department of Physics, Tohoku University, 980 Sendai, Japan \\ T. Mochiku and K. Kadowaki \\ National Research Institute for Metals, Sengen, Tsukuba, Ibaraki 305, Japan
}

(Received 21 February 1996)

\begin{abstract}
Particle-hole $(p-h)$ mixing is a fundamental consequence of the existence of a pair condensate. We present direct experimental evidence for $p$ - $h$ mixing in the angle-resolved photoemission (ARPES) spectra in the superconducting state of $\mathrm{Bi}_{2} \mathrm{Sr}_{2} \mathrm{CaCu}_{2} \mathrm{O}_{8+\delta}$. In addition to its pedagogical importance, this establishes unambiguously that the gap observed in ARPES is associated with superconductivity. [S0163-1829(96)50622-3]
\end{abstract}

A fundamental consequence of the existence of a pair condensate in superconductors is the mixing of particles and holes in the neighborhood of the Fermi energy, $E_{F} \cdot{ }^{1}$ While there are innumerable experiments on superconductors which give indirect evidence for particle-hole $(p-h)$ mixing, it would be very nice, from a pedagogical point of view at least, to have direct experimental evidence for such mixing. Among the conventional probes of superconductivity, Andreev reflection perhaps comes closest to providing a direct test of such mixing, although this is not (usually) a momentum-resolved probe. Here we show that angleresolved photoemission spectroscopy (ARPES) reveals $p$ - $h$ mixing in the most direct and dramatic way: by the appearance of states that do not exist above $T_{c}$.

We have recently provided strong evidence, ${ }^{2}$ by studying sum rules, that the impulse approximation is valid in ultraviolet photoemission studies of the high- $T_{c}$ materials, and that the experimental energy distribution curves (EDC's) can be interpreted ${ }^{3}$ in terms of the one-particle spectral function $A(\mathbf{k}, \omega)$. More specifically, since ARPES measures only occupied states, the intensity is proportional to the Fermi function $f(\omega)$ times $A(\mathbf{k}, \omega)$. Using this we shall study $p$ - $h$ mixing in the superconducting state of $\mathrm{Bi}_{2} \mathrm{Sr}_{2} \mathrm{CaCu}_{2} \mathrm{O}_{8+\delta}$ (Bi2212).

To set the stage for the experimental results it may be useful to recall $p$ - $h$ mixing in the BCS framework (even though there are aspects of the data which are dominated by many body effects beyond weak coupling BCS theory). The BCS spectral function is given by ${ }^{1}$

$$
\pi A(\mathbf{k}, \omega)=\frac{u_{\mathbf{k}}^{2} \Gamma}{\left(\omega-E_{\mathbf{k}}\right)^{2}+\Gamma^{2}}+\frac{v_{\mathbf{k}}^{2} \Gamma}{\left(\omega+E_{\mathbf{k}}\right)^{2}+\Gamma^{2}},
$$

where the coherence factors are $v_{\mathbf{k}}^{2}=1-u_{\mathbf{k}}^{2}=\frac{1}{2}\left(1-\epsilon_{\mathbf{k}} / E_{\mathbf{k}}\right)$ and $\Gamma$ is a phenomenological linewidth. The normal state energy $\epsilon_{\mathbf{k}}$ is measured from $E_{F}$ and the Bogoliubov quasiparticle energy is $E_{\mathbf{k}}=\sqrt{\epsilon_{\mathbf{k}}^{2}+|\Delta(\mathbf{k})|^{2}}$, where $\Delta(\mathbf{k})$ is the gap function. Note that only the second term in Eq. (1), with the $v_{\mathbf{k}}$ coefficient, would be expected to make a significant contribution to the EDC's at low temperatures.

In the normal state above $T_{c}$, the peak of $A(\mathbf{k}, \omega)$ is at $\omega=\epsilon_{\mathbf{k}}$ as can be seen by setting $\Delta=0$ in Eq. (1). We would thus expect to see in ARPES a spectral peak which disperses through zero binding energy as $\mathbf{k}$ goes through $\mathbf{k}_{F}$ (the Fermi surface). In the superconducting (SC) state, the spectrum changes from $\epsilon_{\mathbf{k}}$ to $E_{\mathbf{k}}$ (Fig. 1). As $\mathbf{k}$ approaches the Fermi surface the spectral peak shifts towards lower binding energy, but no longer crosses $E_{F}$. Precisely at $\mathbf{k}_{F}$ the peak is at $\omega=\left|\Delta\left(\mathbf{k}_{F}\right)\right|$, which is the closest it gets to $E_{F}$. This is the manifestation of the gap in ARPES. ${ }^{4-6}$ Further, as $\mathbf{k}$ goes beyond $\mathbf{k}_{F}$, in the region of states which were unoccupied above $T_{c}$, the spectral peak disperses back, receding away 


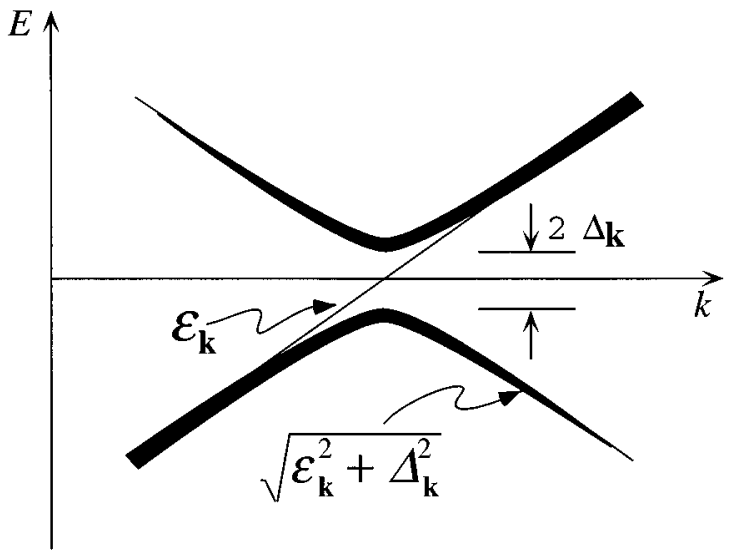

FIG. 1. Schematic dispersion in the normal (thin line) and superconducting (thick lines) states following BCS theory. The thickness of the superconducting state lines indicate the spectral weight given by the BCS coherence factors ( $v^{2}$ below $E_{F}$ and $u^{2}$ above).

from $E_{f}$, although with a decreasing intensity (Fig. 1). This is the signature of particle-hole mixing.

In the remainder of this paper we will present high resolution ARPES data on the high temperature superconductor Bi2212. We shall find that there is clear experimental evidence for the anomalous dispersion described above indicative of $p$ - $h$ mixing. We emphasize that this is the only way (known to us) of asserting that the gap seen by ARPES is due to superconductivity rather than of some other origin, e.g., charge- or spin-density wave formation. Finally, we shall comment on features in the experimental spectra which are determined by many body effects and go beyond the simple BCS expression of Eq. (1).

The results presented below were obtained on the very high quality single crystals $\left(T_{c}=87 \mathrm{~K}\right)$ which were used in our previous Bi2212 studies. ${ }^{2,6,7}$ The measurements were carried out at the University of Wisconsin Synchrotron Radiation Center, using a high resolution $4 \mathrm{~m}$ normal incidence monochromator with a resolving power of $10^{4}$ at $10^{11}$ photons/s. Details about the samples and the experimental procedure may be found in Ref. 6. Even though the momentum window of our spectrometer has a diameter of 0.074 $\AA^{-1}$ at $22 \mathrm{eV}$ photon energy, in this experiment, data were taken at momentum intervals one fourth this value because the spectral peak exhibits a sizable dispersion in the energy and momentum interval of interest to this experiment. As can be seen from the data, the momentum window of the spectrometer does not obscure the dispersion of the spectral peak.

In order to best see $p-h$ mixing one must have a large gap, so that it is better to be near the $\bar{M} Y$ Fermi surface (FS) crossing. $^{5,6}$ [Our notation is $\Gamma=(0,0), \bar{M}=(\pi, 0)$, and $Y=(\pi, \pi)$, where $\Gamma \bar{M}$ is along the $\mathrm{Cu}-\mathrm{O}$ bond direction.] However, the dispersion is very flat in the neighborhood of $\bar{M}$, which makes it hard to establish the bending back of the spectral peaks. On the other hand, while there is significant dispersion in the diagonal $\Gamma Y$ direction, the gap is very small. As a compromise, the data in Fig. 2 are taken along a series of points in momentum space along a path parallel to $\bar{M} Y$ beginning about 0.7 of the way from $\Gamma$ to $\bar{M}$.

First we discuss the normal state $(T=95 \mathrm{~K})$ data shown in Fig. 2(b). Only by contrasting the SC state data with the

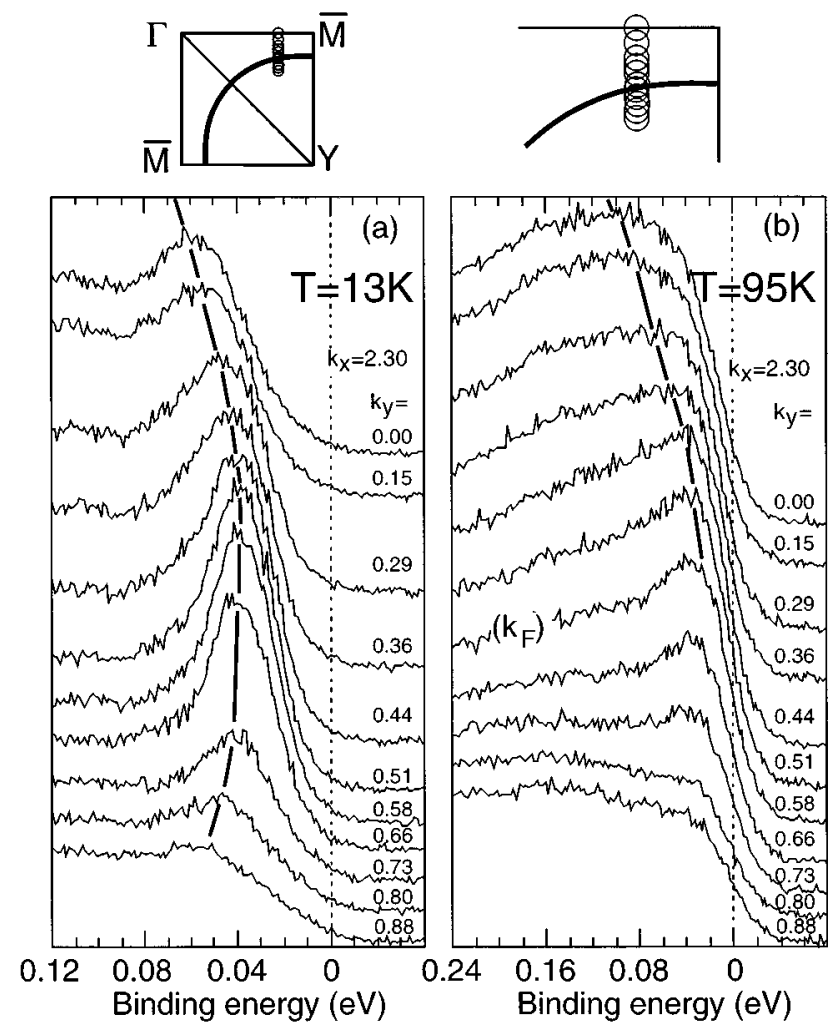

FIG. 2. Superconducting state (a) and normal state (b) EDC's for the same Bi2212 sample for the set of $\mathbf{k}$ values (1/a units) which are shown at the top. Note the different energy ranges.

normal state can one establish $p-h$ mixing. Note that the spectral features are very broad (non-Lorentzian) and asymmetric. The large linewidth is due to many-body effects in the spectral function. The asymmetry, at least in part, comes from the fact that the peak of the EDC corresponds to that of $f(\omega) A(\mathbf{k}, \omega)$, and the Fermi function cuts off what would have been the peak of the spectral function $A(\mathbf{k}, \omega)$. Thus some care is needed in identifying the Fermi surface $\mathbf{k}=\mathbf{k}_{F}$, since it is not a trivial matter to locate the peak of $A(\mathbf{k}, \omega)$ at zero binding energy.

To determine FS location, we use the sum rule ${ }^{2}$ relating the energy-integrated ARPES intensity to the momentum distribution $n(\mathbf{k})$, shown as points in Fig. 3(a) [determined by integrating the normal state data over the range plotted in Fig. 2(b)]. As a background, we used the bottom EDC (0.88) in Fig. 2(b). The error bars are determined mostly by the background subtraction procedure. We then look at the momentum derivative of this integrated intensity, and identify $\mathbf{k}_{F}$ from a peak in $\nabla_{\mathbf{k}} n(\mathbf{k}),{ }^{8}$ a plot of which is shown as a line in Fig. 3(a). From this we see that the EDC labeled 0.58 corresponds to $\mathbf{k}=\mathbf{k}_{F}$. We emphasize that the small peaks seen in the EDC's for $\mathbf{k}$ beyond $\mathbf{k}_{F}$ on the unoccupied side, are not the peaks of the corresponding spectral functions $A(\mathbf{k}, \omega)$, which are presumably at positive binding energy. The EDC peaks come from the Fermi function cutting off $A(\mathbf{k}, \omega)$, as explained above.

We next turn to the superconducting state $(T=13 \mathrm{~K})$ data in Fig. 2(a) at exactly the same set of $\mathbf{k}$ points as the normal state data in Fig. 2(b). The SC state data are plotted on a smaller energy range over which there is a significant in- 

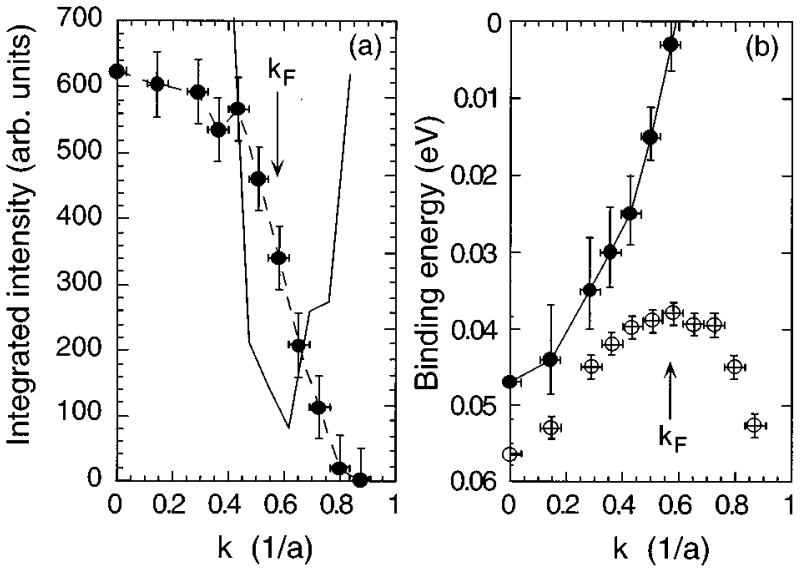

FIG. 3. (a) Integrated intensity versus $\mathbf{k}$ from the normal state data of Fig. 2(b) (black dots), i.e., the momentum distribution, $n_{\mathbf{k}}$. Its derivative is shown as the solid curve (arbitrary scale). The Fermi surface $\left(\mathbf{k}=\mathbf{k}_{F}\right)$ is identified by a peak in the derivative which corresponds within resolution to where $n_{\mathbf{k}}$ is $\frac{1}{2}$. (b) SC state peak positions (white dots) and normal state dispersion (black dots) versus $\mathbf{k}$. Note the backbending of the SC state dispersion for $\mathbf{k}$ beyond $\mathbf{k}_{F}$ which is a clear indication of particle-hole mixing.

crease in the lifetime, i.e., a decrease in the spectral linewidth. Note that the linewidths are smaller than the peak positions relative to $E_{F}$, in complete contrast to the normal state data. The peaks in the superconducting state have resolution-limited widths, that is, one has true quasiparticles in the SC state.

There are three qualitative differences between the normal and SC state data: (1) shift of the spectral feature to lower binding energy due to the opening of a gap; ${ }^{4-6}$ (2) change in line shape due to electron-electron interactions and gap formation; and (3) the change in the dispersion due to $p-h$ mixing. We will briefly discuss the first two points and then turn to the third, which is the main focus of this paper.

First, we would like to demonstrate unambiguously that a gap is indeed present. There have been suggestions in the literature ${ }^{9}$ that one does not see a real gap, since the peak positions of the normal and SC state data do not shift relative to one another as would be expected based on the replacement of $\epsilon_{\mathbf{k}}$ by $E_{\mathbf{k}}$. To refute this argument, we show in Fig. 4 the spectrum at $\mathbf{k}_{F}$ above and below $T_{c}$ in comparison to a reference spectrum of $\mathrm{Pt}$ (in electrical contact with Bi2212) used to establish the Fermi level. One clearly sees that above $T_{c}$, the leading edge slopes of Bi2212 and Pt match. Below $T_{c}$, there is a clear shift of the leading edge of the Bi2212 spectrum to lower binding energy. The apparent match of the peak positions between normal and SC state is just an artifact of the Fermi function cutoff in the normal state which makes the normal state peak appear as if it is below $E_{F}$. We also note that the minimum excitation energy in the SC state occurs at the the same point as the normal state $\mathbf{k}_{F}$ (Fig. 3).

We have previously discussed the changes in the line shape upon cooling through $T_{c}$ in Refs. 2 and 7. We only note here that we do not observe orders of magnitude decrease in the linewidth below $T_{c}$, as microwave experiments ${ }^{10}$ and thermal conductivity ${ }^{11}$ show. This is be-

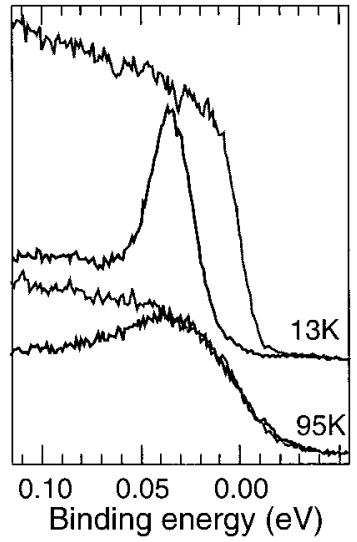

FIG. 4. SC $(T=13 \mathrm{~K})$ and normal state $(T=95 \mathrm{~K}) \mathrm{Bi} 2212$ spectra (solid curves) versus reference Pt spectra (gray curves). Note the clear presence of a gap below $T_{c}$.

cause, as indicated above, the width of our SC state spectra is limited by the energy resolution of our spectrometer.

We now contrast the dispersion of the spectral peaks in the normal and superconducting states of Fig. 2. Above $T_{c}$ the peak of $A(\mathbf{k}, \omega)$ disperses towards the Fermi energy (zero binding energy) and crosses it, as described above in detail. However, in the SC state the same peak is seen to approach the gap value, which is the closest it gets to the Fermi energy, and then recede away from it, with decreasing intensity.

It should be emphasized that the peaks seen in the SC state EDC's for $\mathbf{k}$ beyond $\mathbf{k}_{F}$ are, in fact, the peaks of the corresponding spectral functions $A(\mathbf{k}, \omega)$, in contrast to the normal state. The Fermi function is sharp at $13 \mathrm{~K}$, with a width of order few meV, and the gap has pushed the spectral function down to lower binding energies, thus $f(\omega)$ does not, by itself, produce a peak by cutting off the spectral function, as it did above $T_{c}$.

By comparing the curves for $\mathbf{k}$ beyond $\mathbf{k}_{F}$ in the two panels in Fig. 2, one can see that below $T_{c}$ there are occupied states which did not exist in the normal state. This is a clear signature of particle-hole mixing.

To summarize this, we show in Fig. 3(b) the peak positions of the SC state data versus $\mathbf{k}$ (white dots). The error bars are based on fits of the peak positions after background subtraction. From these fits to the data using Eq. (1) with an appropriate integration over the momentum window and convolutions with the energy resolution and Fermi function, ${ }^{6}$ we have been able to extract off the normal state dispersion (black dots) assuming a SC gap of $33 \mathrm{meV}$ (this gap value being extracted from the spectrum at $\mathbf{k}_{F}$ ). The error bars are asymmetric to account for a possible ( $d$-wave) variation of the gap with $\mathbf{k}$. This dispersion is consistent with the normal state data of Fig. 2. We argue that this is a more accurate method of determination of the normal state dispersion than looking at normal state data, since the latter have poorly defined peak positions due to the large linewidths in the normal state. ${ }^{12}$ Note that the flatness of the SC state dispersion near $\mathbf{k}_{F}$ is reproduced by the fits, arguing that it is a consequence of the finite momentum window rather than some anomalous behavior.

It should be pointed out that while the BCS expression of 
Eq. (1) gives qualitative insight into the observed spectra, the detailed form of the line shape, and its $T$ dependence, arises from many-body physics which is not contained in the simple BCS expression. An example of such a feature is the unusual transfer of spectral weight from the incoherent part of the spectral function to the coherent part upon cooling through $T_{c}$ as seen in Fig. 2. This is most pronounced near $\mathbf{k}_{F}$ where the very broad normal state spectrum (consistent with the absence of a true quasiparticle peak) evolves into a SC state spectrum with a sharp resolution limited peak (rep- resenting a coherent quasiparticle). This is also evident from the $T$-dependent spectra given in Ref. 2.

In conclusion, we have shown that particle-hole mixing in superconductors is directly observable by angle-resolved photoemission spectroscopy.

This work was supported by the U. S. Dept. of Energy, Basic Energy Sciences, under Contract No. W-31-109-ENG38. The Synchrotron Radiation Center is supported by NSF Grant No. DMR-9212658.
${ }^{1}$ See, e.g., J.R. Schrieffer, Theory of Superconductivity (W. A. Benjamin, New York, 1964), p. 122.

${ }^{2}$ M. Randeria et al., Phys. Rev. Lett. 74, 2784 (1995).

${ }^{3}$ In addition there is a more or less nondispersing contribution to the EDC, often called "background." What gives rise to this background is not entirely clear; possible sources are secondary emission due to inelastically scattered electrons, emission from incommensurate superlattice bands known to exist in Bi2212, and emission from disordered $\mathrm{Bi}-\mathrm{O}$ surface layer. We emphasize, however, that the incoherent part of the spectral function is not background.

${ }^{4}$ C.G. Olson et al., Science 245, 731 (1989); Phys. Rev. B 42, 381 (1990).

${ }^{5}$ Z.X. Shen and D.S. Dessau, Phys. Rep. 253, 1 (1995).

${ }^{6}$ H. Ding et al., Phys. Rev. Lett. 74, 2784 (1995); 75, 1425(E) (1995).

${ }^{7}$ H. Ding et al., Phys. Rev. Lett. 76, 1533 (1996).

${ }^{8}$ While a rigorous theoretical justification for this definition (or any other definition at finite temperatures) of $\mathbf{k}_{F}$ is lacking, this method is more general than using $n\left(\mathbf{k}_{F}\right)=1 / 2$ which requires a strong $p-h$ symmetry assumption $A\left(\epsilon_{\mathbf{k}}, \omega\right)=A\left(-\epsilon_{\mathbf{k}},-\omega\right)$. Further, from an experimental point of view, determining the value of the momentum distribution needs both background (Ref. 3) subtraction and knowledge of matrix elements, while the $\mathbf{k}$-derivative method is not affected by either of these, since only $n(\mathbf{k})$ is strongly $\mathbf{k}$ dependent. In practice, the two definitions yield the same $\mathbf{k}_{F}$ within experimental resolution.

${ }^{9}$ K. Miyake and O. Narikiyo, J. Phys. Soc. Jpn. 64, 1040 (1995).

${ }^{10}$ W.N. Hardy et al., Phys. Rev. Lett. 70, 399 (1993).

${ }^{11}$ K. Krishana, J.M. Harris, and N.P. Ong, Phys. Rev. Lett. 75, 3529 (1995).

${ }^{12}$ For energies larger than $3 \Delta$, the linewidth in the SC state becomes comparable to that in the normal state. Therefore, only for energies smaller than $3 \Delta$ can the peak of the spectrum be identified with the quasiparticle binding energy. 